\title{
Remove Unimportant Features from True Colored Images Using the Segmentation Technique
}

\author{
Shahad A. Hasso \\ College of Computing and Mathematics \\ University of Mosul
}

Received on: $24 / 10 / 2010$

Accepted on: 10/11/2010

\section{ABSTRACT}

In this work a new approach was built to apply k-means algorithm on true colored images (24bit images) which are usually treated by researchers as three image (RGB) that are classified to 15 class maximum only. We find the true image as 24 bit and classify it to more than 50 classes. As we know k-means algorithm classify images to many independent classes or features and we could increase the class number therefore we could remove the classes or features that have minimum number of pixels which are considered unimportant features and reconstruct the images.

Correlation factor and Signal to Noise Ratio were used to measure the work and the results seems that by increasing the image resolution the effect of removing minimum features is decreased.

The CSharp (Visual Studio 2008) programming language was used to build the algorithms which are able to allocate huge matrices in high execution time.

Keywords: true colored images, Correlation, Segmentation technique.

إلغاء الصفات غير المهمة من الصور حقيقية الالوان باستخدام تقنية التقيع

$$
\text { كلية عوم الحاسبات والرياضيات حسّو الرحمن }
$$

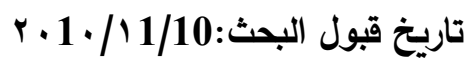

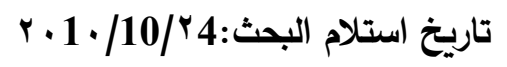

\footnotetext{
الملخص

تم في هذا العمل بناء طريقة جديدة لتطبيق خوارزمية k-means على الصور الملونة الحقيقية (24bit) والتي اعتاد الباحثون عند التعامل معها على تقسيمها الى ثلاثة صور RGB والتي عادة لا يمكن ان يتعدى عدد

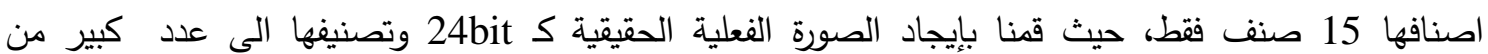
الاصناف تتجاوز الخمسون صنفا وكما نعلم فان خوارزمية k-means تصنف الصور الى عدد كبير من الأصناف او الخصائص مستقلة وحسب مقدار استقلالية كل صنف وبما اننا استطعنا زيادة عدد الاصناف مما مكننا من الغاء الاصناف او الخصائص الاقل عددا من الوحدات الصورية للصورة والتي تعتبر اقل اهمية بالنسبة

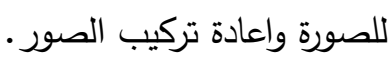

تم قياس تتفيذ العمل باستخدام عامل الارتباط ونسبة الاشاره الى الضوضاء واظههرت النتائج انه كلما ازدادت دقة الصورة (ابعادها) كلما قل تأثير حذف الأصناف غير المهمة منها.
} 
تم تطبيق خوارزميات البحث باستخدام لغة CSharp (Visual Studio 2008) البرمجية والتي توفر

إمكانيات عالية لحجز المصفوفات وكذلك سرعة عالية في التنفيذ. الكلمات المفتاحية: صور حقيقة الألو ان، التصحيح، تقنية التقطيع.

1 - الجة الصور

تهدف معالجة الصور إلى بناء تطبيقات قادرة على فهم محتوى الصور كما يفهمها الإنسان. حيث من

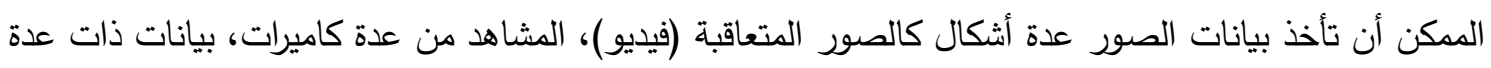
أبعاد مأخوذة من جهاز تصوير طبي. بعض الأمثلة على تطبيقات معالجة الصور [5]:

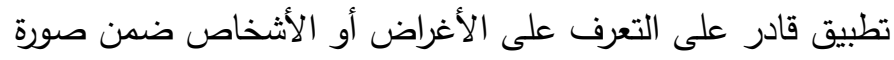
تطبيقات التحكم الآلي (الروبوت والمركبات الآلية). بناء نماذج للأشياء أو للمحيط (الفحص الصناعي، الروبون والمركات الحليل الصورة الطبية).

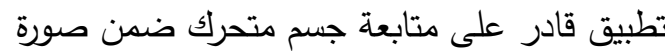

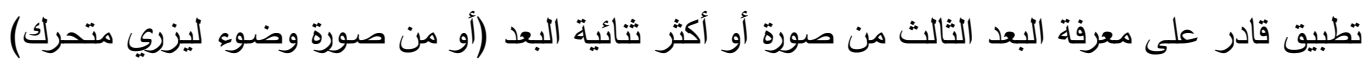

\section{2. 2 مفوم الالوان في الصور الرقمية}

لنموذج اللوني أحمر أخضر أزرق RGB أي (Red Green Blue). وهو نموذج لوني تجمع فيـه الأضواء الحمراء والخضراء والزرقاء مع بعضها البعض بطرق مختلفة لتوليد مجموعة كبيرة من الألوان. الهدف الأساسي من النموذج اللوني RGB هو الإحساس وتوليد وعرض الصور في الأجهزة الإكترونية، مثل شاشات الحواسيب، كان للنظام اللوني RGB نظريـة قويـة خلفه تعتمد على الإدارك البشري للألوان[11]. وتتقم الصور الرقمية بالاعتماد على الألوان إلى ثلاثة أنواع:

2.1 الصور الثنائية (Binary Images):

تمثل الصور الثنائية أبسط أنواع الصور الرقمية، وكل عنصر من عناصر الصورة يمثل قيمة واحدة من قيمتين فقط وتعرض كأبيض وأسود وعدديا فإن القيمتين تمثلان "1" للأبيض و "0" للأسود وتخزن الصور الثنائية كمصفوفة ثنائية الأبعاد من الأصفار والآحاد والصدور الثنائية يطلق عليها عدة تسميات Monochrome) أو (1 Bit Pixel Image) أو (Black And White Image) وذلك لأنها تأخذ رقماً ثنائياً واحداً

لتمثيل كل نقطة[11].

2.2 الصور ذوات التدرج الرمادي (Gray Level Images):

تحتوي الصور ذوات التدرج الرمادي على معلومات إضاءة فقط، ولا توجد فيها معلومات لون، وهذا النوع

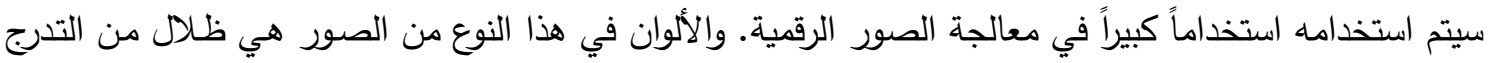

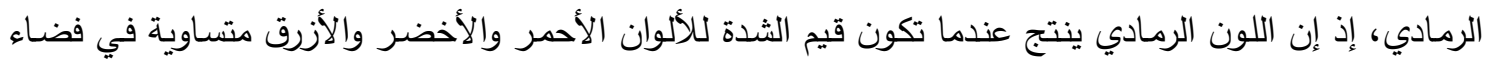
RGB إن عدد الـ الـ Bits المستخدمة لكل نقطة ضوئية يحدد عدد مستويات الإضاءة، وتحتوي الصور المثالية على بيانات (8Bit/Pixel) وتسمح لنا بامتلاك 255-0 من التدرجات الرمادية المختلفة [11]. إن الصور ذوات 
التدرج الرمادي شائعة الاستخدام وذلك بسبب كون الكثير من أجزة العرض واكتساب الصور تستطيع تجهيز صور بـ(8 Bit فضلاً عن ذلك فإن الصور ذات التدرج الرمادي سهلة للعديد من المهام، فلا توجد حاجة إلى استخدام عمليات أصعب وأعقد للمعالجة كما هي الحال بالنسبة للصور الملونة [5].

2.3 الصور الملونة (Color Images):

إن تمثيل الصور الرقمية الملونة يكون بتخصيص قيم شدة منفصلة للألوان الرئيسة الثلاثة، إذ إن اللون

لكل نقطة ضوئية يحدد من خلال تجمع شدات تلك الألوان. وفي حالة خزن الصور الملونة (RGB) بـ(Rits (24 Bits)

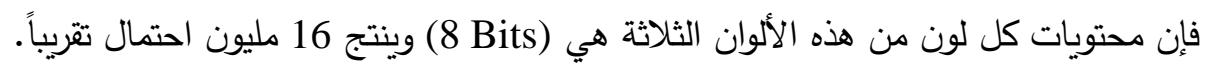

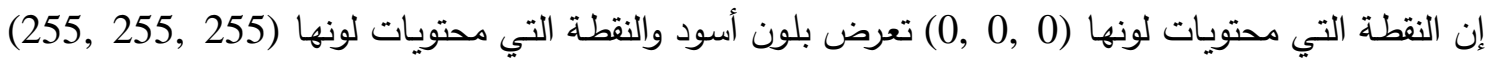
تعرض بلون أبيض، لذلك فإن هذا النوع من الصور يعرف بـ(0) الصور كفوء لتغطية مدى كامل من الألوان التي تدركها العين البشرية إلا أن هناك بعض التصنئ المساوئ في استخدام هذا

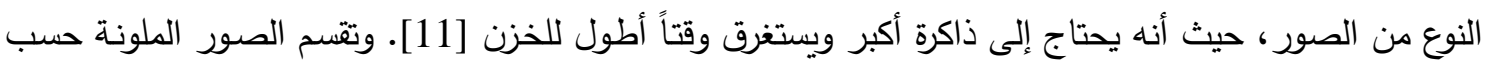
عدد الوانها الى عدة انواع ندرج منها: 1. الصور الملونة ذات المؤشر (256 لون) 2. الصور الملونة ذات 16 بت لون 3. الصور الملونة ذات 24 بت لون 4. الصور الملونة ذات 32 بت لون وفي هذا البحث تم التطرق الى الصور الملونة ذات 24 بت ودراسة خصائصها ومعالجتها 2.3.2 الصور ذات 24 بت لون

وتسمى ايضـا الصور الحقيقية الالوان لان قيم الالوان فيها تعرض بصورة صحيحة على الثاشة وبعدد

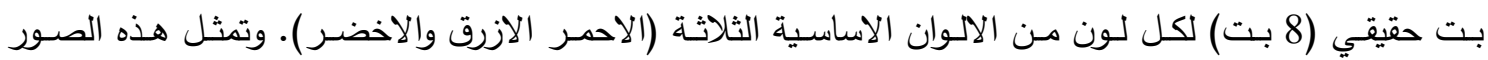

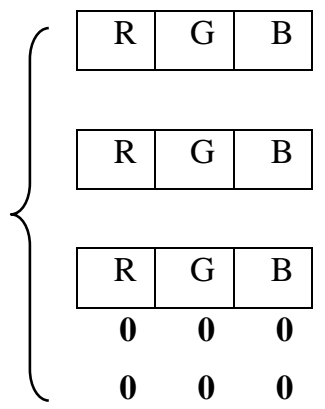

\begin{tabular}{|c|c|c|}
\hline$R$ & $G$ & $B$ \\
\hline$R$ & $G$ & $B$ \\
\hline$R$ & $G$ & $B$ \\
\hline $\mathbf{0}$ & $\mathbf{0}$ & $\mathbf{0}$ \\
$\mathbf{0}$ & $\mathbf{0}$ & $\mathbf{0}$
\end{tabular}

\begin{tabular}{|c|c|c|}
\hline$R$ & $G$ & $B$ \\
\hline$R$ & $G$ & $B$ \\
\hline R & G & B \\
\hline 0 & 0 & 0 \\
0 & 0 & 0
\end{tabular}

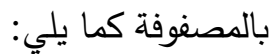

اي ان كل وحدة صورية هي عبارة عن رقم من 24 بت (0-16,777,215) ومن اهم خصـائص هذه الصور انها عالية الدقة وتجانس الالوان فيها يكون كبير جدا مما يجعلها واضحة الرؤيا. ولكن في نفس الوقت فان هذه الصور تحتوي على معلومات غير مهمة ( خصـائص غير مهمة) من المككن ايجادها وحذفها بدون التاثير على الصورة نفسها. 
تقطيع الصور هي مرحلة هامة من مراحل معالجة الصور الرقمية ، وهي عبارة عن عملية تجزئة للصورة إلى مناطق مترابطة ومتجانسة وفقا لمعيار محدد كاللون مثلا، اتحاد هذه المناطق يجب أن ينتج عنه إعادة تكوين الصورة الأصلية. التقطيع مرحلة مهمة تسمح باستخراج معلومات نوعية عن الصورة إذ توفر وصفا عالي المستوى حيث أن كل منطقة مرتبطة بالمناطق المجاورة لها ضمن شبكة من العقد تمثل فيها كل عقدة منطقة في الصورة و تحمل هذه

العقدة بطاقة تحوي معلومات نوعية عن المنطقة كحجمها و لونها و شكلها و توجهها [3].

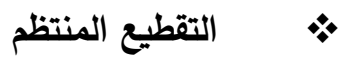

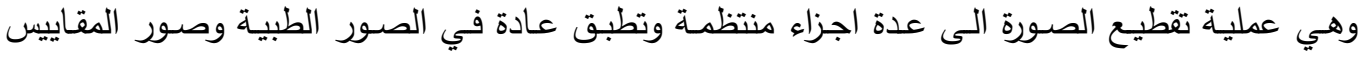
الحيوية حيث يتم قطع الاجزاء غير المهمة حسب احداثيات معينة، وفي هذه العملية تُعتَد احداثيات وابعاد الصور لتحديد عدد القطع وابعادها. والثكل (1) يوضح احد أمثلة التقطيع المنتظم.

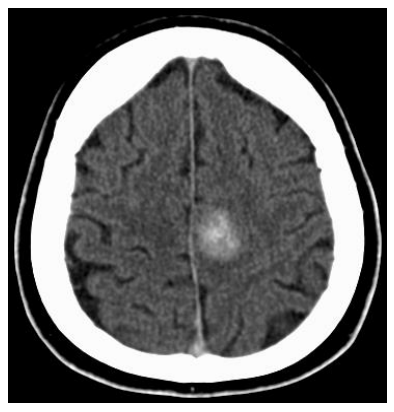

الثكل (1): مثال لصورة بتقطيع منتظم (4×4)

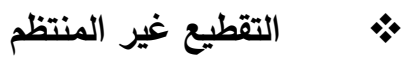

وتسمى ايضا عملية تصنيف الصور وتتم فيها تقطيع الصورة حسب خواصها الى مجاميع غير منتظمة

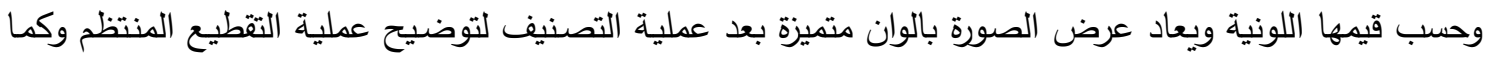
مبين في الثكل (2) والذي يمثل عملية تصنيف صورة خاصة بتطبيقات التحس النائي الى تسعة اصناف][1].

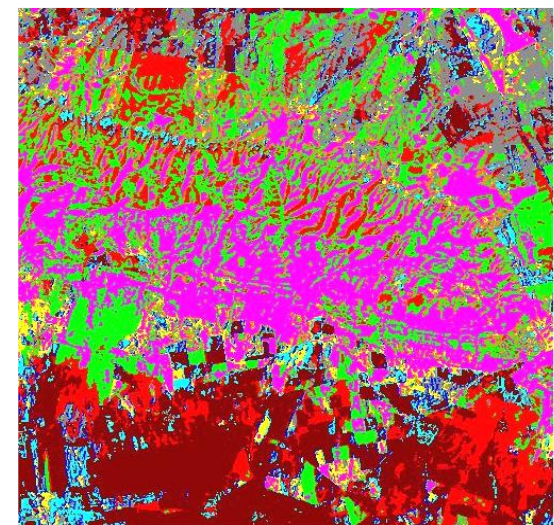

(ب) الصورة بعد اجراء عملية التصنيف عليها

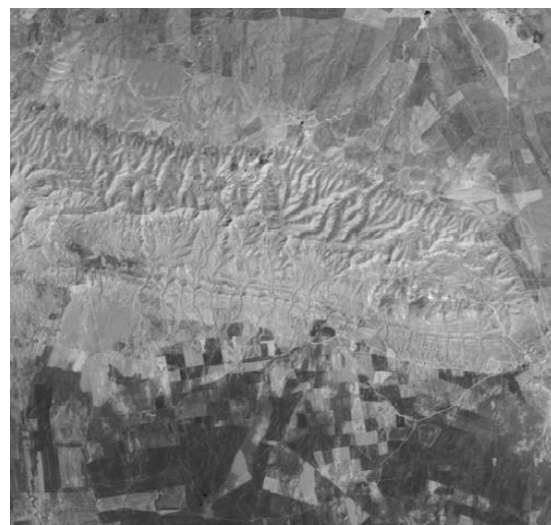

(ا) (الصورة الاصلية

الثكل (2) صورة مصنفة الى تسعة اصناف (تقطيع غير منتظم) 
وكذلك من انواع التقطيع غير المنتظم هو تقطيع الصورة باستخدام انواع من المرشحات Filters ولكل نوع منها

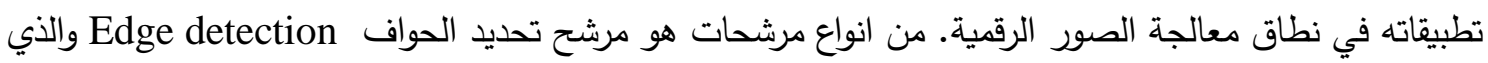

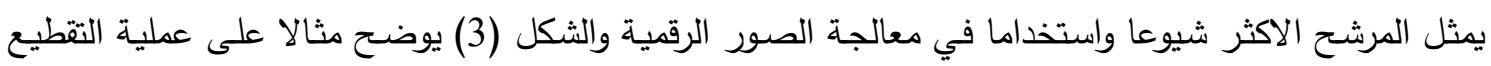
غير المنتظم باستخدام احد مرشحات تحديد الحواف.

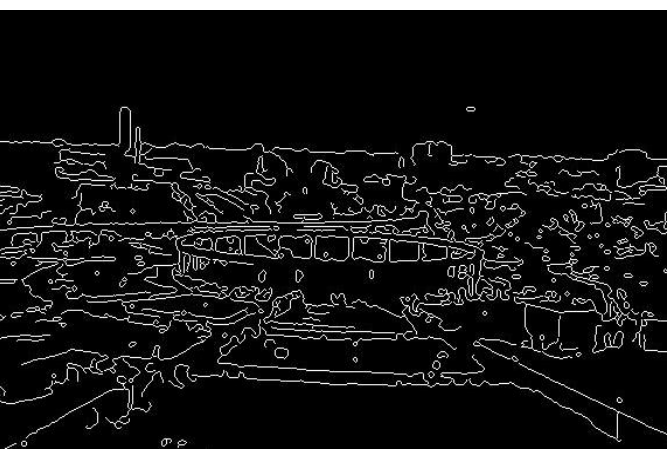

(ب) الصورة بعد تطبيق احد مرثحات تحديد الحواف

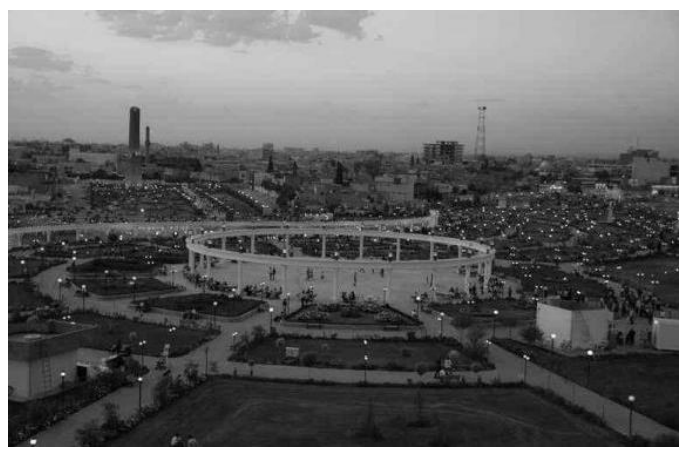

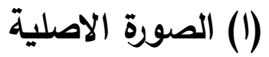

الثكل (3) مثال لصورة بعد تطبيق احد مرشحات تحديد الحواف (تقطيع غير منتظم)

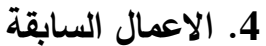

قام عدد كبير من الباحثين بالتطرق الى خوارزميات التصنيف بجميع انواعها وكل منهم قام بتصنيف الصور على اعتبار قيمها تقع بين (225-0) حيث قام الكحلة [1] عام (2004) ببناء نظام للتكامل بين الأسلوب التقليدي والثبكات العصبية في التصنيف غير الموجه لمعطيات التحس النائي، وجاء بعده خروفة [3] في عام

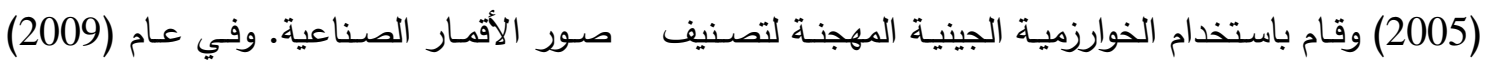

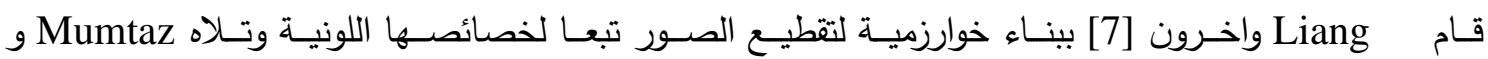
[9] Duraiswamy في (2010) وصمم خوارزمية جديدة لتطوير خوارزمية K-means لتصنيف الصور . 5. 5 ت تصنيف الصور

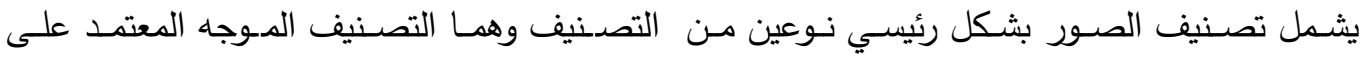

الخصائص الاحصائية للمعطيات والتصنيف غير الموجه المعتمد بثكل رئيسي على درجة التثابه بين الأنماط.

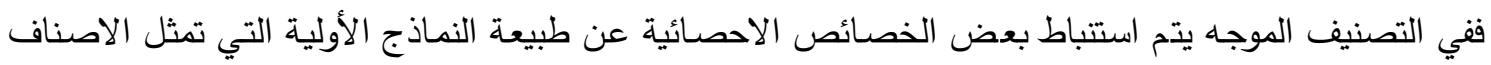

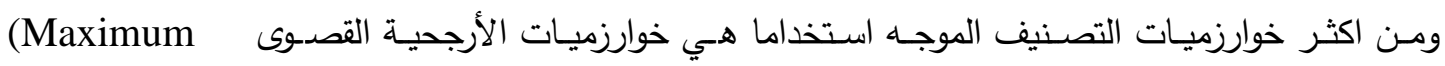

[1] [1] (Minimum Distance Algorithm) وخوارزمية اقصر مسافة Likelihood Algorithm) ففي خوارزمية الأرجحية القصوى يتم حساب مصفوفة التباين والتباين المشترك لكل نموذج في النماذج الداخلة في

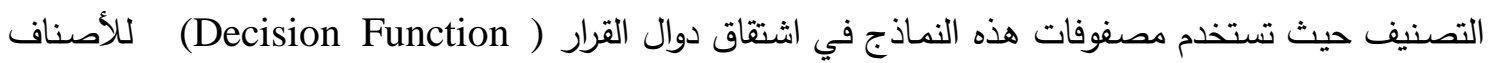
المتوفرة ثم يتم إدخال متجهات النمطية (Pattern Vectors) التي تمثل الخلايا الصورية المراد تصنيفها على فئى دوال القرار ويتم حساب كل دالة ثم مقارنة القيم الناتجة من هذه الدوال وتصنيف المتجه النمطي الداخل تبعا للقيمة القصوى بين قيم الدوال[8]. 
أما في خوارزمية الأقصر مسافة فيتم حساب معدل النماذج الأولية لكل صنف ويتم اشتقاق دوال القرار بالاعتماد

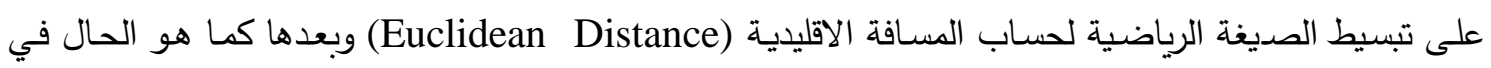
نظرية الأرجحية القصوى يتم تصنيف المعطيات من المرحلة الثانية وذلك بإخخالها بشكل متجهات نمطية على هذه

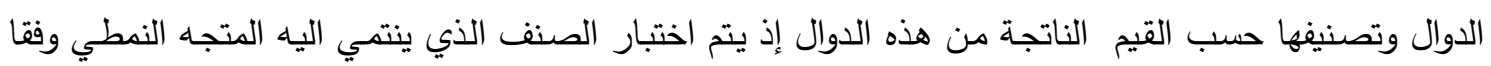
لقيم الدالة، أما في التصنيف غير الموجه يتم حساب درجة التثابه بين المتجهات النمطية الداخلة للتصنيف وتعتبر

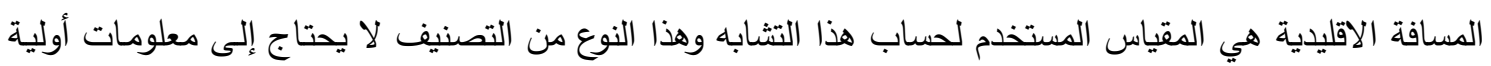
إلا أنها تحتاج إلى تحديد عدد الأصناف مسبقا وأكثر هذه الخوارزميات استخداما هي خوارزمية العنقدة البسيطة (Maxmin Clustring Algorithm) وخوارزميـة أقصى الأدنى هوارنيات (Simple Clustring Algorithm) وخوارزمية (K-means) (11].

\section{6-means خوارزمية}

احدى اهم المعالجات التي تجري على الصور هي عملية تقطيع الصور Segmentation او ما يسىى

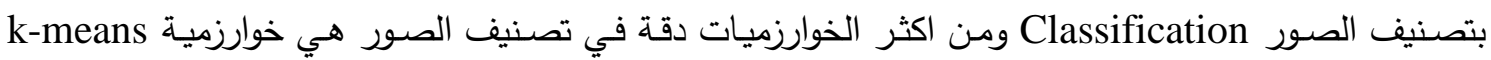
والتي تعطي دقة عالية كونها تبنى على تصحيح المراكز في كل دورة تتفيذ .

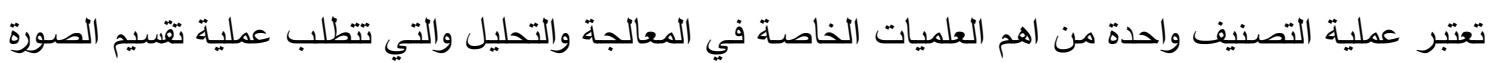

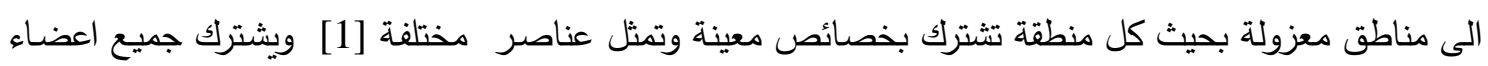

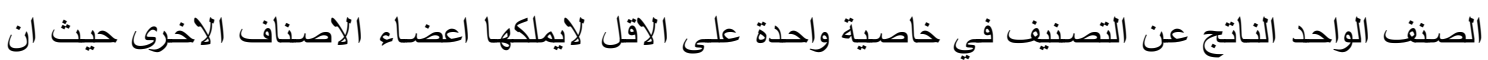

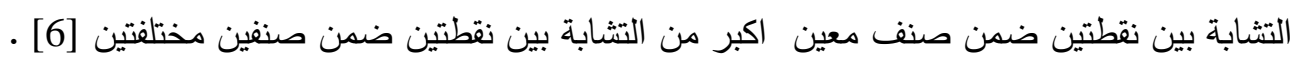

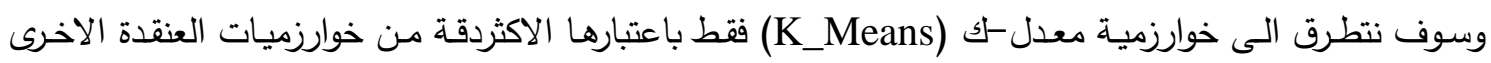

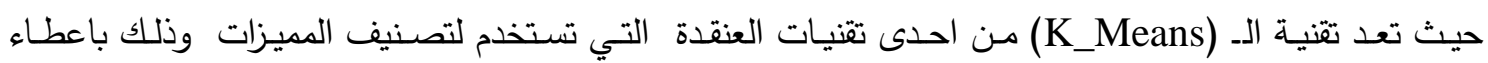

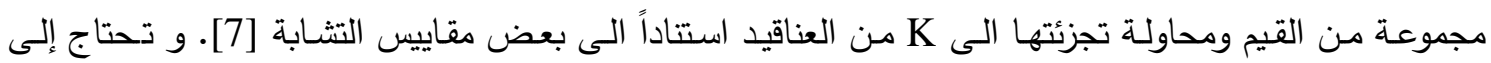

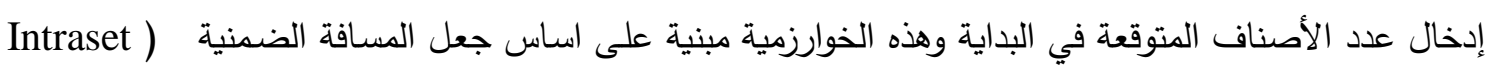

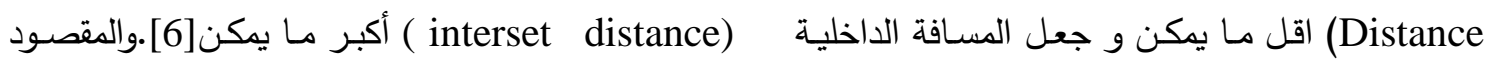
بالمسافة الضمنية intraset distance والمسافة الداخلية interset distance هي ان المسافة الضمنية تمثل مدى التباعد بين انماط الصـفف الواحد وتعتمد دقة التصـيف على اقل مسـافة ضـنية فكلمـا كانت اقل كـان التصنيف ادق اما المسافة الداخليةinterset distance فهي مدى التباعد بين كل صنف عن الاخر وفي هذة

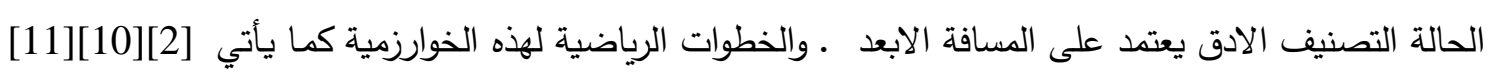

الخطوة الاولى : تحديد عدد الاصناف والمتمثلة بقيمة k. الخطوة الثانية : اختيار مراكز لهذة الاصناف ويتم اختيار هذة المراكز اما بانتقاء نقاط معينة من الصورة بصورة

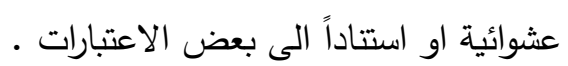

الخطوة الثالثة: حساب المسافة الاقليدية ئية Euclidean distance (Ed) بين نقاط الصورة ومراكز الاصناف وفقا للمعادلة التالية $E d=\left\|Z_{j}(n)-X\right\|$ 


$$
\begin{aligned}
& \text { حيث ان k تمثل عدد الاصناف و } \\
& \text { و x تمثل نقطة الصورة المراد تصنيفها. } \\
& \text { و z تمثل مركز الصنف، n س تمثل رقم الدورة }
\end{aligned}
$$

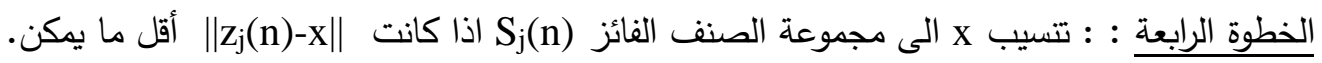

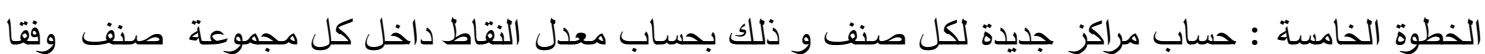
للمعادلة التالية $Z_{j}(n)=1 / N_{j} \sum X i$

حيث ان N تمثل عدد النقاط في المجموعة الخطوة السادسة : قارن بين المراكز القديمة للأصناف (Z) (Z) المحسوبة في الدورة السابقة مع المراكز الجديدة المحسوبة في الدورة الحالية فاذا اختلفت هذه المراكز لصنف واحد على الاقل يعاد حساب الخوارزمية

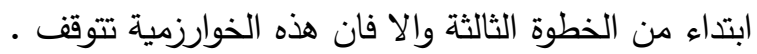
ان خوارزمية ال K_Means تستخدم على نطاق واسع في الكثير من التطبيقات ليس فقط لتصنيف وتتظيم البيانات وانمـا هي مفيده في تمييز الانماطو استرجاع المعلومـات وتحديد الصـوت وكـلام المتكلم وفي تتقيب ميب

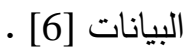

إن من مساوئ هذه الخوارزمية أنها تحتاج إلى وقت طويل وذلك لوجود مرحلة التكرار لتصحيح مراكز الاصناف أما

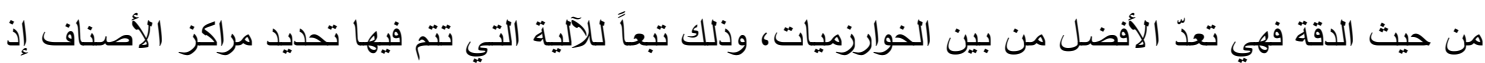

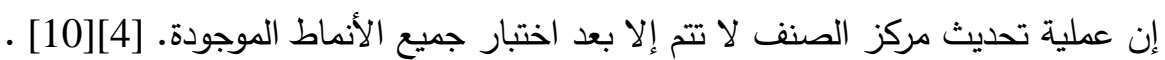

7. - 2. - الخوارزمية المقترحة

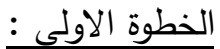
قراءة الصورة الملونة ذات 24 بت كمصفوفة ثلاثية الابعاد يمثل بعدها الاول الالوان الاساسية الثلاثة والبعد الثاني والثالث طول الصورة وعرضها مقاسا بالوحدة الصورية على التوالي الخطوة الثانية:

تحويل الصورة الثلاثية الابعاد الى ثنائية الابعاد مع الاحتفاظ بالقيمة (24 بت) كما هي وحسب المعادلة التالية Img_24bit $[i, j]=\operatorname{img}[0, i, j]$ + img $[1, i, j]$ * 256 + $i=0, \ldots$, width $j=0 \ldots$, height ;

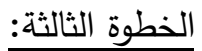

اختيار عدد الاصناف وتطبيق خوارزمية K-means على الصورة مع الاحتفاظ باحداثيات كل نقطة مصنفة الخطوة الرابعة: ترتيب عدد الاصناف تصاعديا والغاء الاصناف الاقل عدد من الوحدات الصورية والتي تمثل خواص قليلة الاهمية في تطبيقات التصنيف الخطوة الخامسة: اعادة تكوين الصورة الناتجة بعد الغاء الخواص غير المهمة 
الخطوة السادسة:

تحويل القيم 24بت الى قيم الالوان الاساسية وحسب المعادلة التالية

Img_out $[0, i, j]=i m g \_24 b i t[i, j]$ \& 256;

Img_out $[1, i, j]=\operatorname{Img} 24 b i t[i, j] \& 65280) / 256$;

$\operatorname{Img}[2, i, j]=\operatorname{Img} 24 \mathrm{bit}[i, j] \&$ 16711680)/ 65536;

حيث تمثل الارقام [ 2, 2 , 0 الالوان الاساسية الثلاثة (RGB) على التوالي

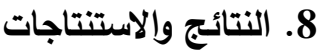

بعد تطبيق الخوارزمية المقترحة على عدد من الصور الملونـة ومـع زيـادة عدد الاصناف قمنا بحساب

عامل الارتباط (correlation factor) ونسبة الاشارة الى الضوضاء (Signal to Noise Ratio) بين الصور المدخلة والصور الناتجة وكما مبين في النتائج المدرجة ادناه:

1- الصورة رقم (1-أ) الصورة الاصلية، الصورة رقم (1-ب) الاصناف الصحذوفة، الصورة رقم (1-جـ) الصورة الناتجة، الجدول على يسار الصورة يمثل عدد الوحدات الصورية لكل صنف مع تحديد الاصناف المحذوفة

\begin{tabular}{|c|c|c|}
\hline & علد الثوحدات & الأصناف ألمذوفة \\
\hline صنف 1 & 2536 & yes \\
\hline صنف2 & 2811 & yes \\
\hline صنف3 & 3996 & yes \\
\hline صنف4 & 5328 & yes \\
\hline صنف5 & 5935 & No \\
\hline صنف6 & 6420 & No \\
\hline صنف7 & 6434 & No \\
\hline صنف8 & 6507 & No \\
\hline صنف9 & 7235 & No \\
\hline صنف 10 & 7412 & No \\
\hline صنف 11 & 7522 & No \\
\hline صنف 12 & 8027 & No \\
\hline صنف 13 & 8304 & No \\
\hline صنف 14 & 9678 & No \\
\hline صنف 15 & 11192 & No \\
\hline صنف 16 & 13257 & No \\
\hline صنف 17 & 13336 & No \\
\hline
\end{tabular}

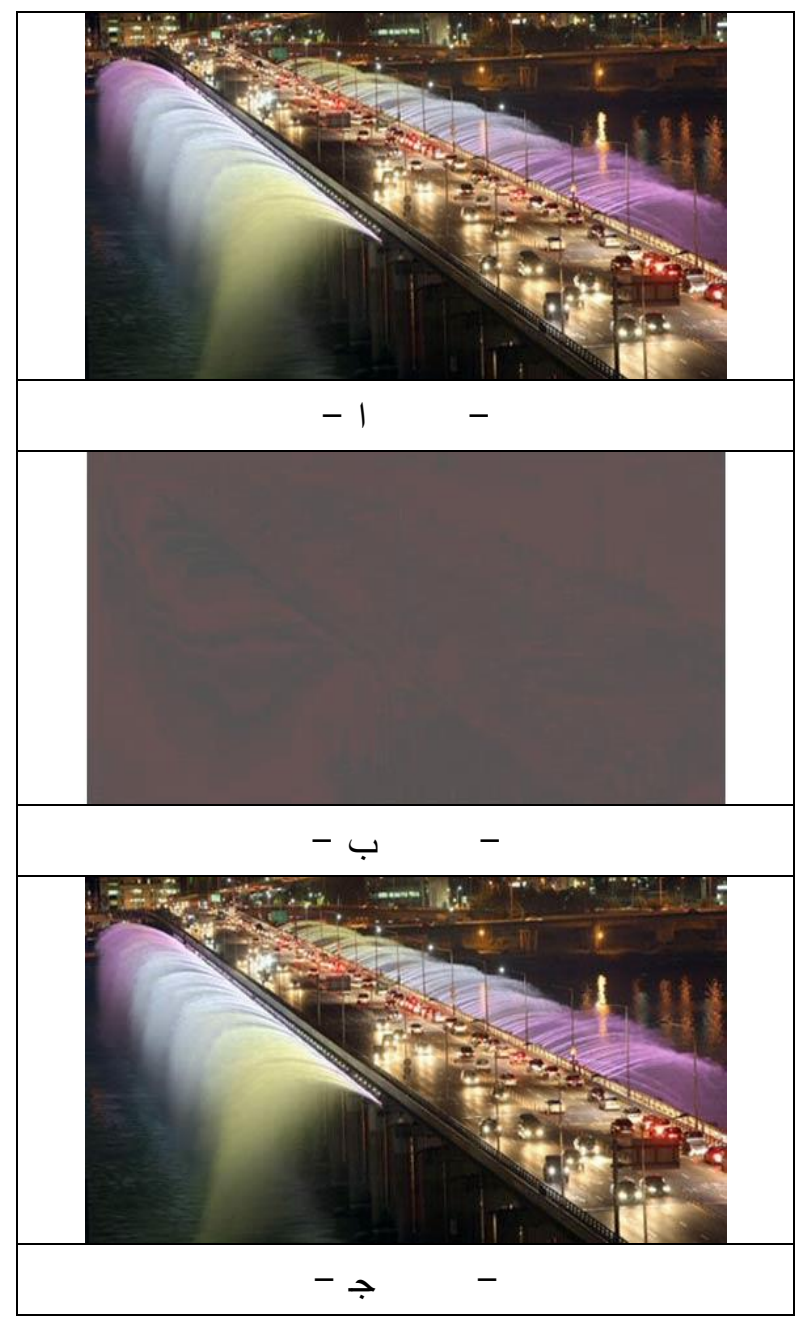

لكل صنف والأصناف المحذوفة عالد الوحلت الصورن 
2- الصورة رقم (2-أ) الصورة الاصلية، الصورة رقم (2-ب) الاصناف الصحذوفة، الصورة رقم (2-ج) الصورة الناتجة، الجدول على يسار الصورة يمثل عدد الوحدات الصورية لكل صنف مع تحديد الاصناف المحذوفة

\begin{tabular}{|c|c|c|}
\hline & علد الوحدات & الاصناف \\
\hline صنف 1 & 7013 & yes \\
\hline صنف2 & 10743 & yes \\
\hline صنف3 & 10863 & yes \\
\hline صنف4 & 10907 & yes \\
\hline صنف5 & 11146 & No \\
\hline صنف6 & 11541 & No \\
\hline صنف7 & 12583 & No \\
\hline صنف8 & 13011 & No \\
\hline صنف9 & 13277 & No \\
\hline صنف 10 & 13617 & $\mathrm{No}$ \\
\hline صنف 11 & 14641 & No \\
\hline صنف 12 & 14778 & No \\
\hline صنف 13 & 15257 & No \\
\hline صنف 14 & 15603 & $\mathrm{No}$ \\
\hline صنف 15 & 158.55 & No \\
\hline صنف 16 & 16529 & No \\
\hline صنف7 17 & 19446 & $\mathrm{No}$ \\
\hline
\end{tabular}

جدول يبين عدد الوحدات الصورية لكل صنف والأصناف المحذوفة

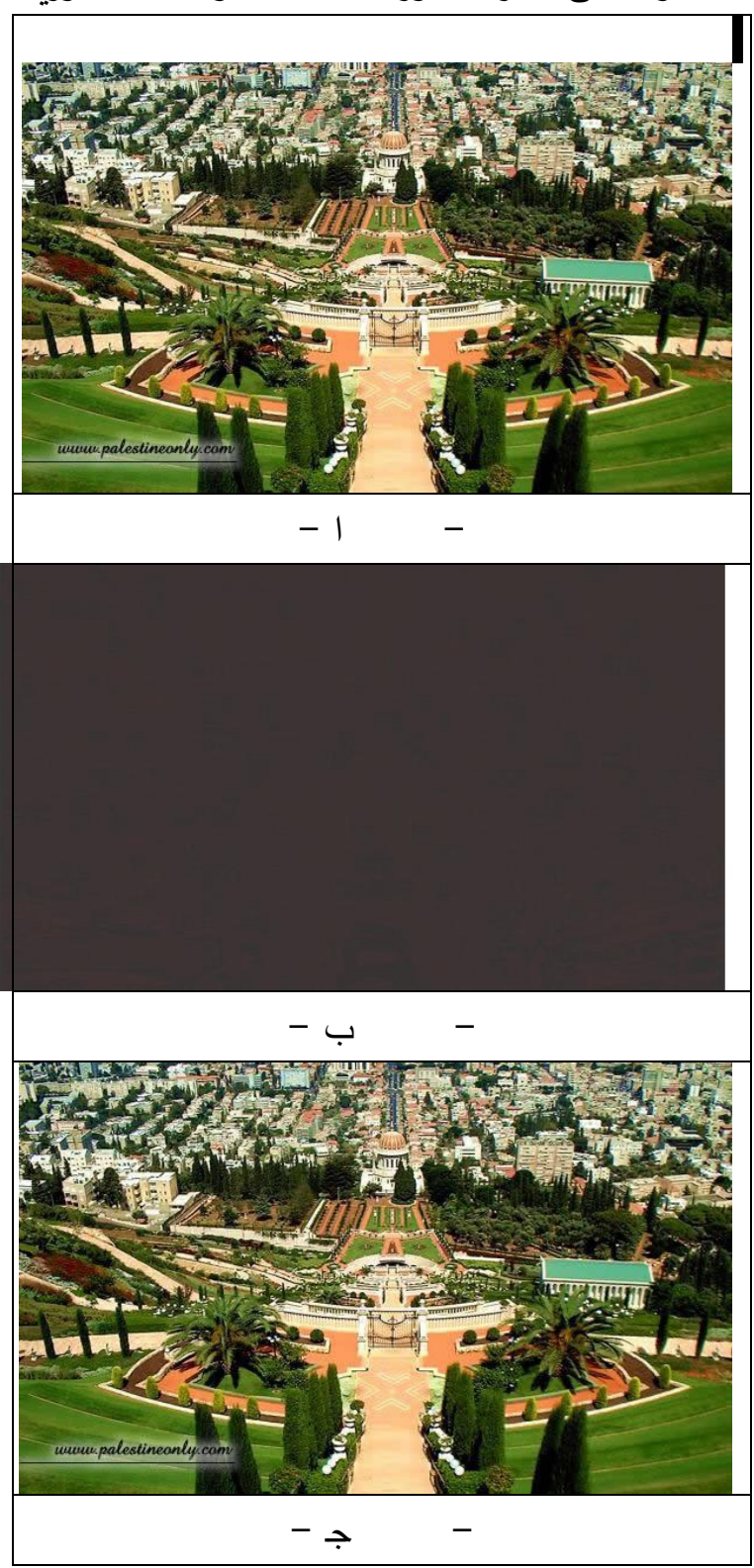




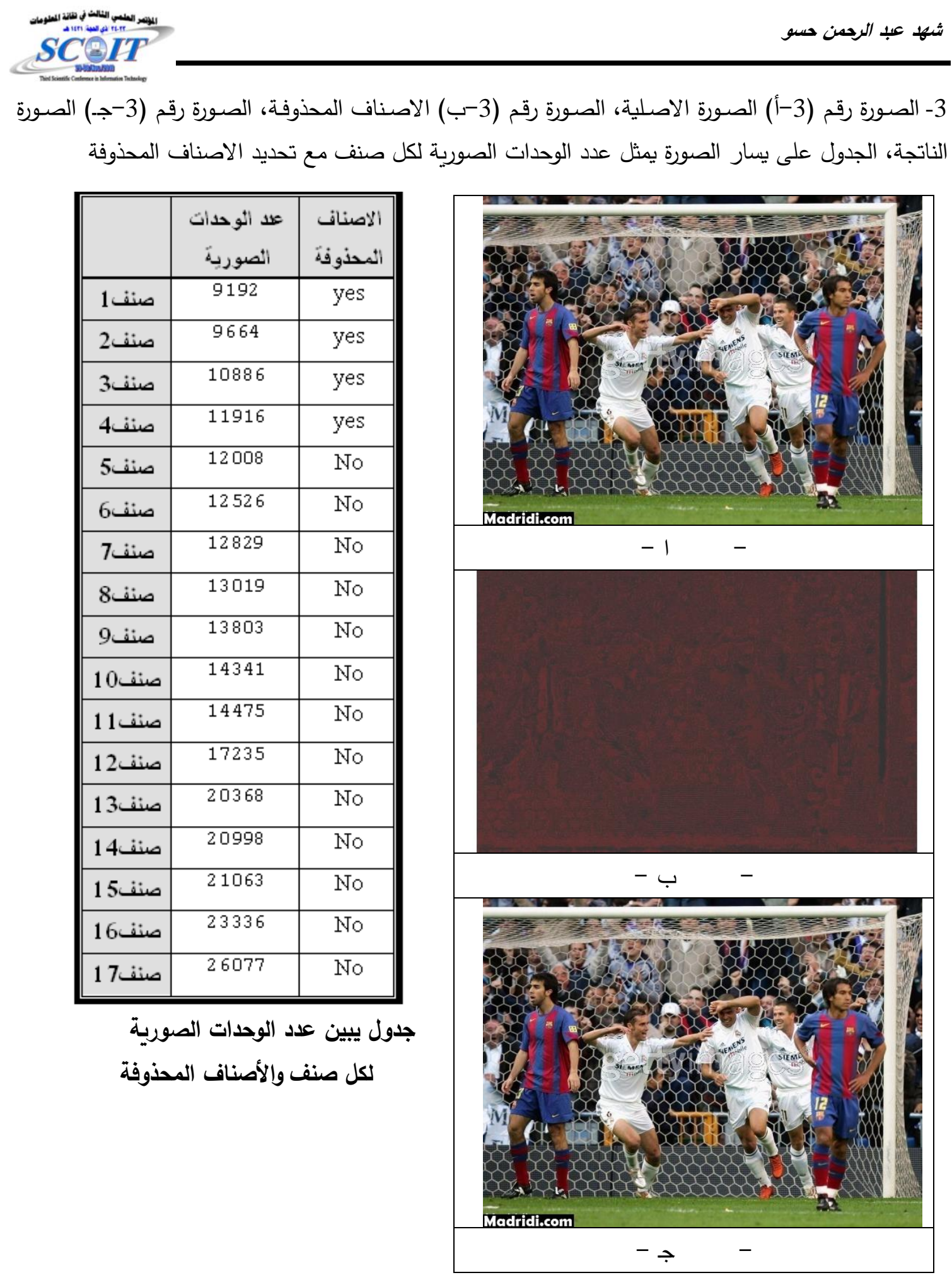

الجدول رقم (1) مقاييس حذف الاجزاء غير المهمة(الاصناف) باختلاف عددها وابعاد الصور (انظر الملحق A). 


\begin{tabular}{|c|c|c|c|c|}
\hline الارنباطد & نسبة الإنسارة & المدد الأصناف & الأصنداف & أبحادورة \\
\hline 0.86 & 70.1021 & 4 & 17 & $\begin{array}{r}\times 490 \\
257\end{array}$ \\
\hline 0.89 & 687805 & 4 & 25 & $\begin{array}{r}\times 490 \\
257\end{array}$ \\
\hline 0.88 & 69.6961 & 8 & 40 & $\begin{array}{r}\times 490 \\
257 \\
\end{array}$ \\
\hline 0.91 & 68.7562 & 10 & 55 & $\begin{array}{r}\times 490 \\
257\end{array}$ \\
\hline 0.936 & 70.8142 & 4 & 17 & $\begin{array}{r}\times 613 \\
370 \\
\end{array}$ \\
\hline 0.911 & 70.9725 & 4 & 25 & $\begin{array}{r}\times 613 \\
370 \\
\end{array}$ \\
\hline 0.92 & 71.4232 & 8 & 40 & $\begin{array}{r}\times 613 \\
370\end{array}$ \\
\hline 0.95 & 71.6548 & 10 & 55 & $\begin{array}{r}\times 613 \\
370\end{array}$ \\
\hline 0.899 & 71.0585 & 4 & 17 & $\begin{array}{r}\times 594 \\
444\end{array}$ \\
\hline 0.921 & 71.9712 & 4 & 25 & $\begin{array}{r}\times 594 \\
444\end{array}$ \\
\hline 0.943 & 71.6377 & 8 & 40 & $\begin{array}{r}\times 594 \\
444\end{array}$ \\
\hline 0.968 & 71.9314 & 10 & 55 & $\begin{array}{r}\times 594 \\
444 \\
\end{array}$ \\
\hline
\end{tabular}

كما هو مبين في الجدول رقم (1) فانه بزيادة أبعاد الصورة يقل تأثير حذف بعض الأصناف على الصور بالرغم

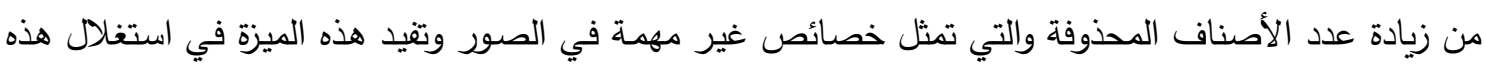
الأصناف في تطبيقات معينة كتطبيقات امنية البيانات وارسالها عبر الثبكات. 


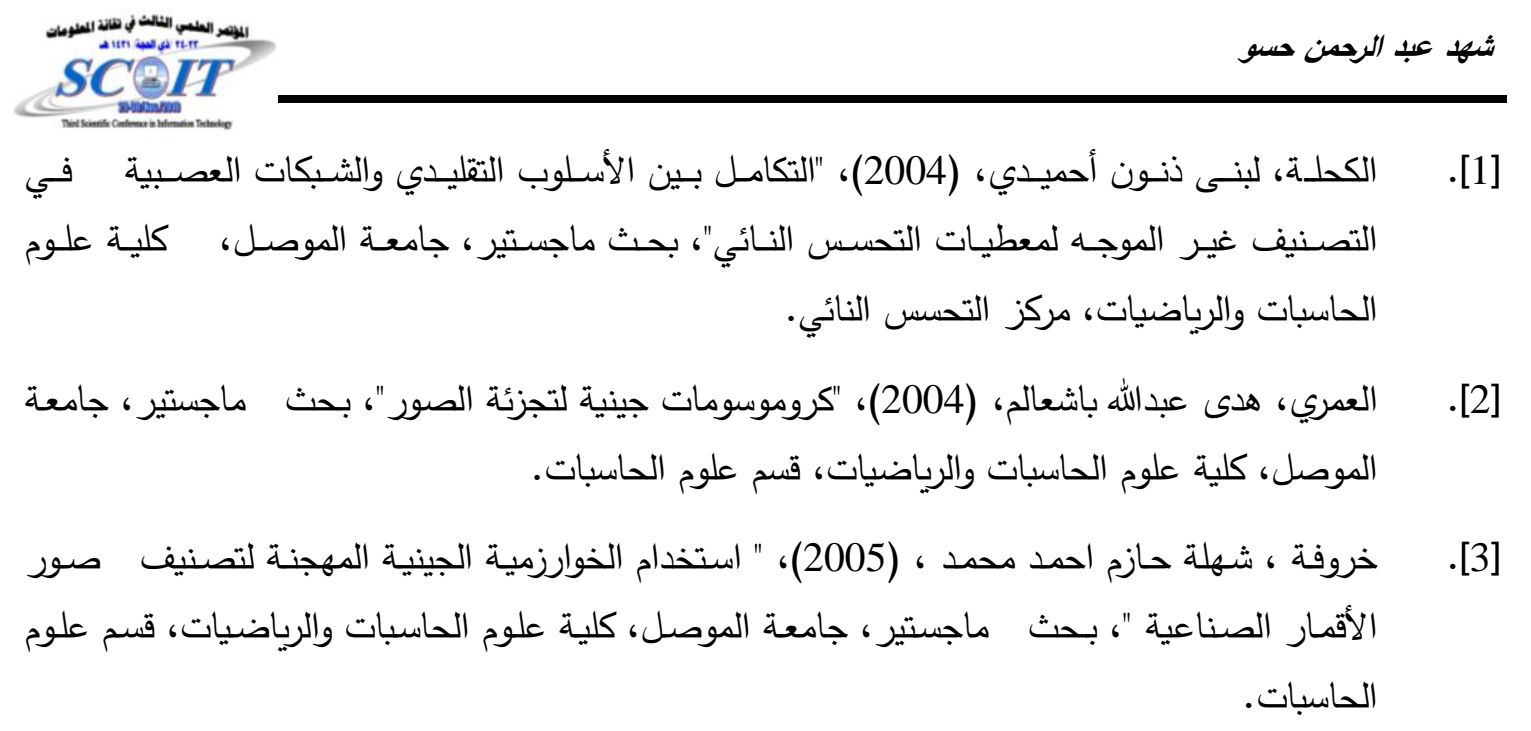

[4]. Chintan A. Shah, Manoj K. Arora and Pramod K. Varshney, (2004),"Unsupervised Classification of Hyperspectral Data: an ICA Mixture Model Based Approach", International journal of Remote Sensing, Vol. 25, No. 2, 481-487.

[5]. Gonzalez, R. C. And Woods, R. E., (2008), "Digital Image Processing", Prentice Hall, Inc., $4^{\text {th }}$ edition.

[6]. Ilea Dana Elena and Whelan Paul F.(2006), "Color image segmentation using a spatial k-means clustering algorithm",.

[7]. Liang Qu, Xinghui Dong and Fadong Guo, (2009), "Automatic K-Means For Color Enteromorpha Image Segmentation", Third International Symposium On Intelligent Information Technology Application, IEEE computer Sociaty, 224 227.

[8]. Mikhled Alfaouri, Khaled Daqrouq, Jamal al-Nabulsi, (2010) ," K-Mean Clustering and Arabic Vowels Formants Based Speaker Identification System" European Journal of Scientific Research, vol.42 no.3, pp.406-417.

[9]. Mumtaz K. and K. Duraiswamy , (2010), "A Novel Density Based Improved KMeans Clustering Algorithm", International Journal on Computer Science and Engineering, India, Vol. 02, No. 02, 213-218 .

[10]. Ravichandran K.S. And Ananthi B., (2009), "Color skin segmentation using kmeans cluster", International Journal of Computational and Applied Mathematics, india volume 4 number 2 pp. 153-157.

[11]. Umbaugh, Scott E., (1998), "Computer Vision And Image Processing”, Prentice Hall PTR, USA. 


\begin{tabular}{|c|c|c|c|c|c|c|c|c|c|}
\hline \multirow{2}{*}{\multicolumn{10}{|c|}{ 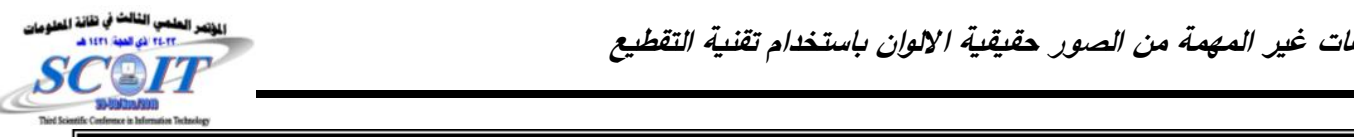 }} \\
\hline & & & & & & & & & \\
\hline \multicolumn{3}{|c|}{ 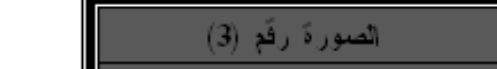 } & \multicolumn{3}{|c|}{ ألصورة رَّج (2) } & \multicolumn{3}{|c|}{ 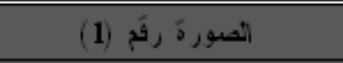 } & \multirow{3}{*}{ تُشنف } \\
\hline \multicolumn{3}{|c|}{ عدد الاصناف } & \multicolumn{3}{|c|}{ عدد الاصنافت } & \multicolumn{3}{|c|}{ عدد الاصناف } & \\
\hline 55 & 40 & 25 & 55 & 40 & 25 & 55 & 40 & 25 & \\
\hline 1164 & 2432 & 4453 & 1122 & 22225 & 2105 & 371 & 850 & 1254 & 1 \\
\hline 1218 & 2522 & 4624 & 1736 & 2388 & 4317 & 820 & 1002 & 2181 & 2 \\
\hline 1288 & 2733 & 4967 & 1779 & 2430 & 4658 & 832 & 1453 & 3486 & 3 \\
\hline 2211 & 3375 & 5415 & 2017 & 2610 & 5424 & 841 & 1454 & 3703 & 4 \\
\hline 2437 & 3793 & 5964 & 2101 & 2665 & 5825 & 1187 & 1480 & 4004 & 5 \\
\hline 2528 & 3900 & 6205 & 2105 & 2739 & 7824 & 1253 & 1534 & 4016 & 6 \\
\hline 2563 & 3905 & 6948 & 2216 & 2775 & 7912 & 1286 & 1572 & 4480 & 7 \\
\hline 2582 & 3913 & 7749 & 2271 & 3440 & 8121 & 1306 & 1763 & 4932 & 8 \\
\hline 2733 & 4178 & 8541 & 2302 & 3463 & 8265 & 1313 & 1790 & 5050 & 9 \\
\hline 2897 & 4506 & 8994 & 2346 & 3709 & 9119 & 1403 & 1843 & 5066 & 10 \\
\hline 3153 & 4710 & 11442 & 2553 & 3722 & 9368 & 1529 & 1848 & 5069 & 11 \\
\hline 3206 & 5069 & 12031 & 2684 & 4197 & 9374 & 1555 & 1905 & 5148 & 12 \\
\hline 3288 & 5083 & 12036 & 2726 & 4281 & 9547 & 1561 & 1913 & 5428 & 13 \\
\hline 3368 & 5110 & 12149 & 2878 & 4424 & 9645 & 1593 & 1968 & 5475 & 14 \\
\hline 3485 & 5349 & 12460 & 2896 & 4658 & 9867 & 1774 & 2150 & 5496 & 15 \\
\hline 3518 & 5966 & 12674 & 2994 & 4703 & 9926 & 1851 & 2248 & 5605 & 16 \\
\hline 3678 & 6177 & 12755 & 3015 & 4972 & 10311 & 1855 & 2264 & 5675 & 17 \\
\hline 3788 & 6189 & 13183 & 3051 & 5085 & 10313 & 1884 & 2331 & 5776 & 18 \\
\hline 3878 & 6338 & 13320 & 3152 & 5169 & 10661 & 1953 & 2516 & 5856 & 19 \\
\hline 3934 & 6529 & 13678 & 3182 & 5298 & 11092 & 1975 & 2553 & 5906 & 20 \\
\hline 3998 & 6583 & 13932 & 3192 & 5346 & 11289 & 1989 & 3007 & 5920 & 21 \\
\hline 4026 & 7016 & 14152 & 3221 & 5417 & 11693 & 1993 & 3078 & 6088 & 22 \\
\hline 4096 & 7020 & 14186 & 3294 & 5419 & 13024 & 1993 & 3597 & 6365 & 23 \\
\hline 4189 & 7133 & 15407 & 3341 & 6142 & 13099 & 2020 & 3679 & 6521 & 24 \\
\hline $\begin{array}{l}4247 \\
4332\end{array}$ & $\begin{array}{l}7182 \\
7309\end{array}$ & 16771 & $\begin{array}{l}3459 \\
3511\end{array}$ & $\begin{array}{l}6240 \\
6395\end{array}$ & 14031 & $\begin{array}{l}2065 \\
2094\end{array}$ & $\begin{array}{l}3786 \\
3810\end{array}$ & 7430 & $\begin{array}{l}25 \\
26\end{array}$ \\
\hline $\begin{array}{l}4395 \\
4470\end{array}$ & $\begin{array}{l}7532 \\
7571\end{array}$ & & $\begin{array}{l}3558 \\
3708\end{array}$ & $\begin{array}{l}6539 \\
6555\end{array}$ & & $\begin{array}{l}2176 \\
2197\end{array}$ & $\begin{array}{l}3934 \\
3936\end{array}$ & & $\begin{array}{l}27 \\
28\end{array}$ \\
\hline $\begin{array}{l}4710 \\
4862\end{array}$ & $\begin{array}{l}7747 \\
7751\end{array}$ & & $\begin{array}{l}4196 \\
4426\end{array}$ & $\begin{array}{l}6583 \\
7023\end{array}$ & & $\begin{array}{l}2281 \\
2290\end{array}$ & $\begin{array}{l}4043 \\
4125\end{array}$ & & $\begin{array}{l}29 \\
30\end{array}$ \\
\hline 4903 & 7796 & & 4553 & 7377 & & 2310 & 4278 & & 31 \\
\hline 5038 & 7969 & & 4658 & 8174 & & 2355 & 4417 & & 32 \\
\hline 5161 & 8124 & & 4694 & 8216 & & 2366 & 4426 & & 33 \\
\hline 5195 & 8279 & & 4705 & 8265 & & 2425 & 4531 & & 34 \\
\hline 5240 & 8491 & & 4747 & 8292 & & 2524 & 4654 & & 35 \\
\hline 5416 & 10141 & & 4814 & 9274 & & 2536 & 4781 & & 36 \\
\hline 5422 & 10798 & & 4901 & 9315 & & 2560 & 5083 & & 37 \\
\hline 5442 & 10904 & & 5008 & 9613 & & 2669 & 5920 & & 38 \\
\hline 5612 & 12876 & & 5030 & 10831 & & 2736 & 6978 & & 39 \\
\hline 5901 & 13737 & & 5088 & 10841 & & 2815 & 7430 & & 40 \\
\hline 5977 & & & 5093 & & & 2849 & & & 41 \\
\hline 5984 & & & 5272 & & & 2962 & & & 42 \\
\hline 5992 & & & 5418 & & & 2974 & & & 43 \\
\hline 6102 & & & 5420 & & & 3077 & & & 44 \\
\hline 6133 & & & 5431 & & & 3192 & & & 45 \\
\hline 6411 & & & 5479 & & & 3288 & & & 46 \\
\hline 6780 & & & 5498 & & & 3292 & & & 47 \\
\hline 6793 & & & 5552 & & & 3401 & & & 48 \\
\hline 6940 & & & 6292 & & & 3561 & & & 49 \\
\hline 7327 & & & 6454 & & & 3618 & & & 50 \\
\hline 8037 & & & 7031 & & & 3764 & & & 51 \\
\hline 8105 & & & 7106 & & & 3785 & & & 52 \\
\hline 9131 & & & 7560 & & & 3786 & & & 53 \\
\hline 10104 & & & 7906 & & & 3849 & & & 54 \\
\hline 10348 & & & 8068 & & & 3996 & & & 55 \\
\hline
\end{tabular}

